\section{Determinantes das patentes em ciências da vida e da saúde nas universidades federais de Minas Gerais, Brasil: uma análise de dados em painel para o período 1995-2016}

\author{
Determinants of patents in the life sciences and \\ health sciences at federal universities in Minas \\ Gerais State, Brazil: an analysis of panel data for \\ 1995-2016
}

\section{Determinantes de las patentes en el ámbito de las ciencias de la vida y la salud en universidades federales de Minas Gerais, Brasil: un análisis de datos de panel durante el período 1995-2016}

Inaiara Cóser 1

Marcos Antônio Fernandes Brandão 1

Nádia Rezende Barbosa Raposo 1

Eduardo Gonçalves 1

doi: 10.1590/0102-311X00097517

\section{Resumo}

Na década de 1990, com o desenvolvimento da microeletrônica e das tecnologias de comunicações, emergiu o paradigma da tecnologia da informação, caracterizado pela intensidade de informação que, transformada em conhecimento e inovação, desempenha um papel crucial para o desenvolvimento. Entretanto, para que isso ocorra, é necessário que os países construam arranjos institucionais apropriados. Com isso, surge o modelo de sistemas de inovação da Tríplice Hélice, que compreende a inovação e o progresso técnico, em uma Economia do Conhecimento, como o resultado de um conjunto complexo de relações entre universidade, empresa e governo. Nesse contexto, o presente estudo tem o objetivo de identificar o impacto da produção de conhecimento e das politicas públicas para a produção de depósitos de patentes dos pesquisadores pertencentes ao domínio científico Ciências da Vida e da Saúde, no âmbito das universidades federais de Minas Gerais, Brasil. A análise considerou o período de 1995 a 2016, e utilizou técnicas de econometria em painel de dados. Os resultados mostram que fatores educacionais (orientações em pósgraduação), econômicos (produtos tecnológicos e prestação de serviços tecnológicos) e políticos (arcabouço legal, recursos financeiros e políticas de estímulo) impactam a produção de depósitos de patentes dos pesquisadores selecionados. Por meio da análise dos resultados, podem-se perceber os esforços do governo, mesmo que ainda incipientes, na tentativa de promover o salto tecnológico.

Atividades Científicas e Tecnológicas; Tecnologia da Informação;

Patentes; Inovação
Correspondência

I. Cóser

Rua Santos Dumont 506, apto. 405, Juiz de Fora,

MG 36010-386, Brasil.

inaiaracoser@gmail.com

1 Universidade Federal de Juiz de Fora, Juiz de Fora, Brasil. 


\section{Introdução}

A adoção de novas tecnologias ocorre como resultado de uma interação complexa e iterativa entre desenvolvedores, usuários, agentes reguladores e sociedade civil 1. Após uma inovação radical dar origem ao aparecimento de um novo produto, capaz de gerar uma nova indústria, há um período inicial de inovação intensiva e de otimização, até que o produto seja incorporado pelo mercado ${ }^{2}$. Essas inovações provocam fricções e flutuações em todo o sistema econômico, conduzindo-o, inicialmente, a uma situação de expansão e prosperidade e, posteriormente, a crise e depressão - parte do processo de rejuvenescimento intermitente do sistema econômico - que recriam as condições para um novo ciclo 3 .

Cada mudança de paradigma não só modifica o ambiente de negócios, mas também o contexto institucional e até mesmo cultural e social. Por isso, são necessários novas regras e regulamentos, formação especializada e outros facilitadores institucionais ${ }^{4}$. As novas dinâmicas introduzidas no sistema de produção ocasionam maior ruptura na evolução anteriormente existente nos mercados, transformando gradualmente o tecido social e tornando os mecanismos institucionais obsoletos e contraproducentes.

A revolução tecnológica é confrontada com a resistência de indivíduos, empresas e instituições, que muitas vezes permanecem embutidos no paradigma anterior 5. Sendo assim, os padrões de ação devem ser entendidos também em termos comportamentais, visto que as inovações, que ocorrem ao longo do tempo, surgem por meio de um processo social, de aprendizagem individual e coletiva 6,7 . Países que possuem uma estrutura institucional necessária ou que buscam construir os novos arranjos apropriados para o novo paradigma são os que alcançam sucesso de crescimento e ingressam em um processo de convergência e aproximação com o país líder tecnologicamente.

No contexto do atual paradigma, caracterizado pelo volume de informação, o conhecimento é o principal insumo para a geração de inovação e, consequentemente, para impulsionar crescimento e desenvolvimento econômico ${ }^{8,9}$. No contexto em que o conhecimento é considerado o mais importante recurso para o desenvolvimento e a aprendizagem, o processo mais estratégico 10, a pesquisa acadêmica, assume importância econômica, pois cria oportunidades tecnológicas que devem ser aproveitadas pelos setores industriais para a criação e a utilização de inovação 11 .

Na busca de tentar entender as políticas de Ciência, Tecnologia e Inovação (CT\&I) e gerar estratégias alternativas para o crescimento econômico e a transformação social, Etzkowitz \& Leydesdorff 12 formularam o conceito de Tríplice Hélice. Esse modelo de sistema de inovação propõe que a capacidade de uma nação de gerar conhecimento e convertê-lo em riqueza e desenvolvimento social depende da ação articulada de três principais atores: empresa, universidade e governo 13. Esse modelo assume que a força motriz do desenvolvimento econômico é a produção e a disseminação do conhecimento socialmente organizado. Por isso, as universidades são consideradas tradicionalmente como fonte principal de inovações e mudança tecnológica.

Diante desse contexto, as patentes têm ganhado grande notoriedade para a configuração de políticas públicas nas discussões sobre o papel das universidades em um sistema caracterizado pela produção de conhecimento capaz de gerar inovações. Como resultado desse processo, vários países, entre eles o Brasil, adotaram novas legislações que influenciaram a função das instituições de ensino e pesquisa, no que se refere à proteção do conhecimento. Como forma de analisar a produção de conhecimento tecnológico, os dados de patentes têm a vantagem de representar o resultado do processo inventivo, possuindo aplicabilidade industrial e algum grau de novidade, além de serem facilmente acessíveis e permitirem algum grau de comparabilidade entre pesquisadores, regiões e países. Em que pesem as vantagens relativas ao uso de dados de patentes como proxy da inovação ou atividade tecnológica, é preciso ressaltar que há possível subestimação da atividade tecnológica, pois nem todas as inovações são patenteadas e, em alguns casos, há ausência de valor econômico relevante de algumas patentes 14 . No entanto, como não há medida perfeita, segue-se a literatura internacional, que considera as patentes como medidas críveis da atividade tecnológica 15.

Enfim, o presente estudo teve como objetivo identificar os determinantes dos depósitos de patentes, por características individuais (senioridade acadêmica, produção de conhecimento e efeito fixo do pesquisador) dos pesquisadores mineiros da área de Ciências da Vida e da Saúde, além de verificar se as políticas públicas influenciaram esse patenteamento, representadas pelas dummies de tempo. A análise considerou o período de 1995 a 2016, utilizando técnicas de econometria em painel de dados. 
Este artigo segue a tradição de outros, que analisaram o processo de patenteamento a partir de seus determinantes, como Schettino et al. 16 e Calderini et al. 17, que focaram no inventor individual, avaliando fatores determinantes de produtividade, qualidade de patentes e relação entre produção de artigos versus patentes. Também segue Gurmu et al. 18, que avaliaram a produção de patentes por universidades, e Baldini et al. 19, que analisaram as motivações e obstáculos para patenteamento de professores universitários italianos. Em relação aos trabalhos anteriores, este estudo traz evidências da realidade nacional, em particular do Estado de Minas Gerais, Brasil, para algumas das questões levantadas, como possível conflito entre produção científica versus produção tecnológica, evolução do patenteamento na área de Ciências da Vida e da Saúde e sua possível relação com as políticas públicas adotadas no contexto brasileiro.

\section{Tríplice Hélice}

O modelo da Tríplice Hélice está centrado na infraestrutura de conhecimento das inovações fornecidas pelas relações entre empresa, universidade e governo 20. Ao desenvolverem o modelo, Etzkowitz \& Leydesdorff 13 definiram três estágios distintos: (i) Tríplice Hélice I - o Estado envolve o meio acadêmico e a indústria, além de dirigir as relações entre eles; (ii) Tríplice Hélice II - as esferas institucionais são separadas, possuem fronteiras fortes que as dividem, e as relações entre elas são altamente circunscritas; (iii) Tríplice Hélice III - apresenta uma sobreposição das esferas institucionais, o que consiste em uma infraestrutura de conhecimento, com cada um interagindo com o papel do outro e com organizações híbridas que emergem em relação às interfaces.

Esses três estágios representam uma mudança na dinâmica da sociedade, que se inicia com um limite forte entre as esferas institucionais e organizações, para um sistema de sobreposição mais flexível e dinâmico, com cada um fazendo o papel do outro. Etzkowitz \& Leydesdorff 13 concluíram que o estágio I é visto como um modelo ultrapassado de desenvolvimento, ao passo que o estágio II implica uma política de Laissez-faire, defendida como terapia de choque para reduzir o papel do Estado no estágio I. A Tríplice Hélice, representada pelo estágio III, ocorre quando os três atores entram em uma relação recíproca, na qual cada um tenta melhorar o desempenho do outro 21.

A partir da transferência de conhecimento entre as esferas institucionais, surgem as oportunidades para a aprendizagem mútua e a cooperação que, se não houver perda de identidade, estimulam a criação de novos conhecimentos e, ao mesmo tempo, contribuem para ampliar a capacidade inovativa 22,23. Entretanto, para estimular o processo de inovação, é necessário criar novas relações entre os atores e instigar maior confiança entre eles 24.

Vista como um modelo analítico, a Tríplice Hélice contribui para a descrição da variedade de arranjos institucionais e modelos políticos 13. No entanto, essa reestruturação exige a participação do governo, com o intuito de mudar as condições institucionais 25 e assegurar que os mecanismos econômicos e políticos não sejam vistos como de controle, mas como mecanismos de feedback seletivos que permitem o desenvolvimento do conhecimento científico e tecnológico 26 . Para isso, devem-se levar em consideração os padrões evolutivos vindos de tendências históricas e culturais que influenciam os mecanismos de política pública 6 .

\section{Políticas públicas de estímulo à inovação}

Em termos de diretrizes estabelecidas para a política de inovação, Koeller 27 ressalta que, apesar das ações esparsas, o período de 1999-2002 foi marcado pelo início da articulação de um discurso pró-política de inovação. No entanto, pode-se dizer que a inovação no Brasil passou a ter maior amplitude a partir de 2003, com a criação da Lei de Inovação.

A aprovação da Lei de Inovação em 2004 representou um novo paradigma para o estímulo à inovação no Brasil 28. A Lei Federal no 10.97329 de 02 de dezembro de 2004, regulamentada pelo Decreto no 5.563 de 11 outubro de 2005, dispôs sobre incentivos à inovação e à pesquisa científica e tecnológica no ambiente produtivo e apresentou estímulo à interação entre universidades e empresas, com o apoio à construção dos Núcleos de Inovação Tecnológica (NIT). Em seu artigo 16, estabeleceu 
que toda instituição de Ciência e Tecnologia deverá dispor de NIT, próprio ou em associação, com a finalidade de gerir sua política de inovação 29. Para Arbix \& Consoni 30 (p. 207), essa lei "gerou uma inflexão na trajetória do sistema de gestão da propriedade intelectual e de transferência de tecnologia na universidade brasileira".

A criação desse arcabouço legal ampliou a participação do Estado no apoio à inovação, principalmente por meio da concepção de programas de financiamento com juros subsidiados, concessão de recursos subvencionados às empresas para o custeio de atividades de pesquisa e inovação e contratação de pesquisadores. Acrescentam-se a concessão de incentivos fiscais para a inovação tecnológica e o apoio a fundos de capital de risco para empresas iniciantes 28.

As políticas de estímulo à inovação, apesar de incipientes, demonstram o esforço do governo em criar um arcabouço legal e uma infraestrutura para absorção e desenvolvimento de tecnologias e inovação. Entretanto, muitos têm sido os desafios para a promoção da interação entre universidades e empresas, sendo necessárias não apenas políticas nacionais, mas políticas estaduais.

\section{Políticas de estímulo à inovação em Minas Gerais}

Em consonância com o governo federal, principalmente a partir da Lei de Inovação nacional, o Estado de Minas Gerais também passou a implementar políticas públicas de incentivo à inovação, com o intuito de alavancar a competitividade das empresas mineiras. A principal política de estímulo à inovação foi a elaboração do Plano Mineiro de Desenvolvimento Integrado (PMDI) 2007-2023, em que foram estabelecidas estratégias para o desenvolvimento de longo prazo do estado. Dentre essas estratégias, a criação de empresas dinâmicas e inovadoras aparece como um dos principais destinatários das políticas públicas 31 .

Uma das ações estabelecidas no PMDI foi o projeto estruturador rede de inovação tecnológica, criado pela Secretaria de Estado de Ciência, Tecnologia e Ensino Superior em 2007. O objetivo da rede de inovação tecnológica era ampliar a capacidade de inovação tecnológica do setor produtivo, por meio da dinamização do sistema mineiro de inovação. Para isso, foram estabelecidas cinco linhas de atuação 32: (1) apoio à criação, ampliação e gestão de incubadoras e parques tecnológicos; (2) efetivação do arcabouço legal da inovação, que culminou na elaboração e promulgação da Lei Mineira de Inovação, a criação do Fundo de Incentivo à Inovação Tecnológica e a criação e o fortalecimento da Rede Mineira de Propriedade Intelectual; (3) suporte aos processos de inovação e empreendedorismo tecnológico, que envolveu a criação e implementação do Programa de Incentivo à Inovação (PII) e o apoio à criação dos NIT nas instituições de pesquisa mineiras; (4) fomento à cultura empreendedora, com foco em metodologias de ensino e conteúdos sobre empreendedorismo para jovens e adultos; (5) articulação de parcerias e interações estratégicas entre universidade, empresa e governo, promovida pelo portal Sistema Mineiro de Inovação (SIMI), e a efetivação de parcerias para execução colaborativa de políticas públicas.

Dentre as várias políticas, o PII tem sido abordado como um importante instrumento capaz de favorecer as iniciativas trilaterais descritas pelo modelo da Tríplice Hélice, em que empresas e universidades operam em prol da inovação, ainda que em escala incipiente 33 .

O PII tem o objetivo de transformar a pesquisa universitária em produtos inovadores, por meio da transferência de tecnologia (licenciamentos) ou da geração de novas empresas criadas para explorar a propriedade intelectual, as chamadas spin-offs. Desde sua criação em 2007 até 2010, tinham sido realizados 11 PIIs em nove instituições de ensino/pesquisa mineiras, dentre elas as universidades federais de Lavras, Itajubá, Juiz de Fora, Viçosa, Minas Gerais e Uberlândia. Como resultados tangíveis do PII, Gonçalves \& Cóser 33 destacaram a geração de 88 produtos tecnológicos, 58 patentes, a criação de 15 spin-offs, o licenciamento de quatro tecnologias e a transferência de 17 resultados de pesquisa universitária. Além desses, os autores argumentaram sobre a importância do programa para acelerar o processo de conversão do conhecimento científico em inovação, de forma a contribuir para a construção de uma cultura empreendedora nas universidades mineiras. 


\section{Metodologia}

A base de dados utilizada foi construída a partir de três fontes: sítio da Fundação de Amparo à Pesquisa do Estado de Minas Gerais (FAPEMIG), Plataforma Lattes do Conselho Nacional de Desenvolvimento Científico e Tecnológico (CNPq) e sítio do Instituto Nacional de Propriedade Industrial (INPI). A Figura 1 demonstra o esquema para a amostragem e a construção do banco de dados.

A partir da definição da amostra, por meio do software ScriptLattes (http://scriptlattes.sourcefor ge.net/) 34, foram obtidos os dados referentes à produção de conhecimento de cada pesquisador, no período de janeiro de 1975 a 31 de agosto de 2016. Os dados dos depósitos de patentes foram obtidos de forma manual, no sítio do INPI, em novembro de 2016. As variáveis são retratadas no Quadro 1.

Por usar o Currículo Lattes como fonte de produção dos pesquisadores, as proxies deste artigo podem refletir as limitações dessa base. Ainda que de forma imprecisa, o Lattes permite captar inserção social, econômica, científica e educacional do trabalho dos pesquisadores 35 . Assim, as variáveis foram distribuídas em quatro indicadores de impacto, conforme Quadro 2.

Foi utilizada a análise de dados em painel para identificar os determinantes dos depósitos de patentes dos pesquisadores, e foi empregado o programa estatístico Stata, versão 13 (StataCorp LP, College Station, Estados Unidos), para a modelagem econométrica.

Os modelos de regressão com dados em painel agregam uma combinação de séries temporais e de observações em corte transversal, multiplicadas por T períodos de tempo, o que permite analisar relações significativas entre as variáveis dependentes e explicativas, quer ao longo do tempo, quer entre os indivíduos a serem analisados 36 .

\section{Figura 1}

Esquema para construção do banco de dados.

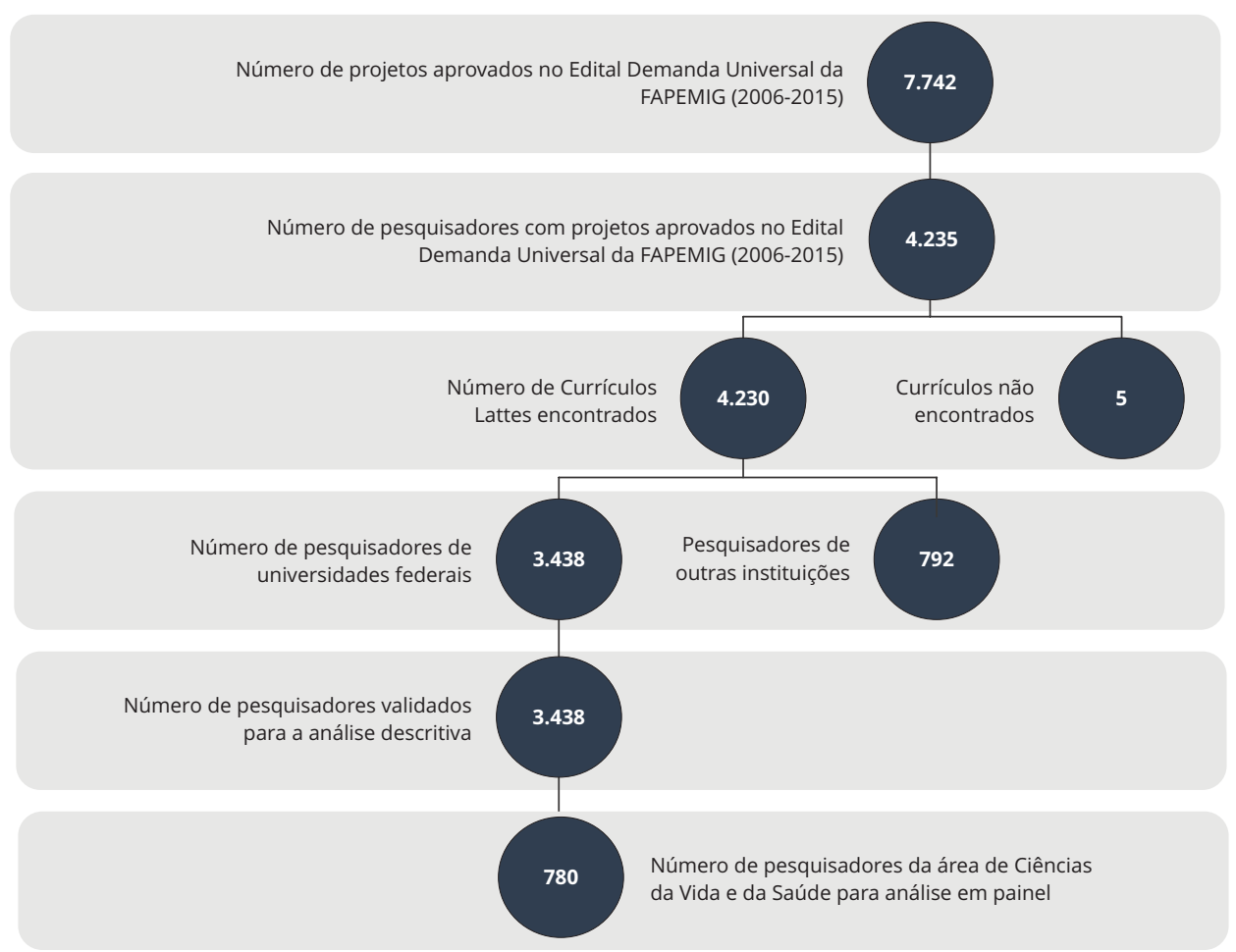

FAPEMIG: Fundação de Amparo à Pesquisa do Estado de Minas Gerais.

Fonte: elaboração própria. 


\section{Quadro 1}

Variáveis de interesse relacionadas à produção de conhecimento e de tecnologia.

\begin{tabular}{|c|c|c|}
\hline Variáveis & Descrição & Fonte \\
\hline SEN & $\begin{array}{c}\text { Senioridade acadêmica do pesquisador. É calculada com base no total de anos em que cada } \\
\text { pesquisador teve, pelo menos, uma produção científica (artigo, livro ou capítulo de livro), desde o ano } \\
\text { de publicação da primeira produção. }\end{array}$ & CNPq \\
\hline DOMÍNIO CIENTÍFICO & $\begin{array}{l}\text { Domínio científico do pesquisador: Ciências da Vida e da Saúde; Ciências Exatas e da Engenharia; } \\
\text { Ciências Sociais e Humanidades; Ciências Naturais e do Ambiente } 46 .\end{array}$ & CNPq \\
\hline ORI & $\begin{array}{c}\text { Número de orientações de Iniciação Científica, Graduação e outras. Foram consideradas as } \\
\text { orientações concluídas e em andamento. }\end{array}$ & CNPq \\
\hline ORIP & $\begin{array}{l}\text { Número de orientações de Pós-Doutorado, Doutorado e Mestrado. Foram consideradas as } \\
\text { orientações concluídas e em andamento. }\end{array}$ & CNPq \\
\hline ART & Número de artigos em periódicos. & CNPq \\
\hline LIV & Número de livros e de capítulos de livros. & CNPq \\
\hline CON & Número de trabalhos completos, resumos expandidos e resumos. & CNPq \\
\hline TEC & $\begin{array}{l}\text { Número de trabalhos técnicos, produção técnica e processos e técnicas. A produção técnica engloba } \\
\text { assessoria, consultoria, parecer, elaboração de projeto, relatório técnico, serviços na área da saúde, } \\
\text { apresentação de trabalho, cursos de curta duração, desenvolvimento de material didático, editoria, } \\
\text { organização de evento, programa de rádio e TV e relatório de pesquisa. }\end{array}$ & CNPq \\
\hline PRO & Número de produtos tecnológicos. & CNPq \\
\hline ARTE & Número de produções artísticas. & CNPq \\
\hline JOR & Número de textos em jornais, artigos em revistas (não periódicos) e outras produções bibliográficas. & CNPq \\
\hline PAT & Número de depósitos de patentes. & INPI \\
\hline
\end{tabular}

CNPq: Conselho Nacional de Desenvolvimento Científico e Tecnológico; INPI: Instituto Nacional de Propriedade Industrial.

Fonte: elaboração própria.

Ao utilizar dados em painel, há muito mais informação para se estudar o fenômeno e graus de liberdade adicionais. Pode-se destacar, como principal vantagem dessa técnica, a ênfase da heterogeneidade individual, por sugerir a existência de características diferenciadoras dos indivíduos, as quais podem ou não ser constantes ao longo do tempo. Ainda, segundo Marques 37, a análise em painel permite tipificar as respostas de diferentes indivíduos a determinados acontecimentos, em momentos distintos. No entanto, no modelo de dados em painel, podem ocorrer problemas relacionados ao enviesamento de seleção, isto é, erros resultantes da seleção dos dados que não formam uma amostra aleatória. Para que as estimativas geradas pudessem ser consistentes e não enviesadas, os efeitos das variáveis não observadas foram considerados na regressão.

Foram estimados sequencialmente o modelo de dados agrupados por MQO (mínimos quadrados ordinários - do inglês OLS - ordinary least squares), o modelo de efeitos aleatórios e o modelo de efeitos fixos. Após a estimação por efeitos aleatórios, realizou-se o teste de Breusch-Pagan para detectar a presença/ausência de efeitos não observados. O resultado, por sua vez, foi significativo a $1 \%$, permitindo a rejeição da hipótese nula de ausência de efeitos não observados. Nesse caso, considera-se que os pesquisadores analisados possuem características individuais não observadas (heterogeneidade não observada, como maior habilidade ou capacidade inventiva), as quais podem influenciar na produção de patentes. A escolha do método foi pautada no teste de Hausman, que rejeitou a hipótese nula, na qual os efeitos aleatórios são consistentes para identificar os fatores não observados. Logo, foi selecionada a modelagem por efeitos fixos.

Para definir os fatores determinantes da produção de patentes, foram utilizadas as variáveis senioridade e as relativas à produção de conhecimento dos 780 pesquisadores da área das Ciências da 


\section{Quadro 2}

Indicadores das principais formas de produção acadêmica.

\begin{tabular}{|l|c|c|c|}
\hline Impacto & Publicações & Outras formas \\
\hline Educacional & Livros-texto, ementas de cursos, textos didáticos & $\begin{array}{c}\text { Formação de pesquisadores qualificados } \\
\text { (p.ex.: mestres e doutores) }\end{array}$ & Arquivos de dados \\
\hline Científico & Artigos em periódicos científicos, livros e capítulos em livros & ORI e ORIP \\
\hline Econômico & Patentes, relatórios de pesquisa contratada & Produtos, processos, software, spin-off & TEC, PRO e ARTE \\
\hline Social & $\begin{array}{c}\text { Guias profissionais, artigos em jornais, comunicações } \\
\text { submetidas a mídias sociais (incluindo blogs) }\end{array}$ & $\begin{array}{c}\text { Entrevistas, participação em eventos, } \\
\text { consultoria científica }\end{array}$ \\
\hline
\end{tabular}

Fonte: adaptado de Moed \& Halevi 35.

Vida e da Saúde. Por meio da dummy de tempo, foi possível analisar o impacto das políticas públicas de CT\&I na produção de depósitos de patentes. A análise foi baseada no modelo econométrico representado pela Equação 1:

$$
\begin{aligned}
\mathrm{PAT}_{i t}= & \alpha_{i t}+\beta_{1} \mathrm{ORI}_{i t}+\beta_{2} \mathrm{ORIP}_{i t}+\beta_{3} \mathrm{ART}_{i t}+\beta_{4} \mathrm{LIV}_{\mathrm{it}}+\beta_{5} \mathrm{CON}_{i t}+\beta_{6} \mathrm{TEC}_{i t}+\beta_{7} \mathrm{PRO}_{i t}+ \\
& \beta_{8} \mathrm{ARTE}_{i t}+\beta_{9} \mathrm{JOR}_{i t}+\beta_{10} \mathrm{SEN}_{i t}+u_{i t}(i=1, \ldots, 780 ; \mathrm{t}=1995, \ldots, 2016)
\end{aligned}
$$

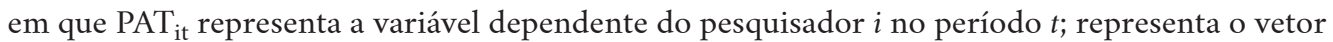
de efeitos específicos para cada pesquisador; $\beta_{1}$ a $\beta_{10}$ são os parâmetros a serem estimados; ORI, ORIP, ART, LIV, CON, TEC, PRO, ARTE, JOR, SEN são as variáveis explicativas; $u$ representa o termo de erro.

\section{Resultados}

Com base nos testes realizados, foram analisados os fatores determinantes da produção de 726 patentes de 780 pesquisadores mineiros selecionados. Os resultados encontram-se na Tabela 1.

A variável senioridade (SEN) não apresentou significância estatística, o que indica que os anos de produção científica do pesquisador não interferiram na produção de depósitos de patentes (terceira coluna, da Tabela 1).

Com relação às variáveis de impacto educacional (ORIP e ORI), apenas a variável orientações em pós-graduação (ORIP) foi significativa (nível de 5\%) e positivamente relacionada à produção de depósitos de patentes. Assim, pode-se dizer que há uma associação positiva entre o número de orientações de pós-graduação e os depósitos de patentes no INPI.

Todas as variáveis com impacto científico (livros, congressos e artigos) apresentaram sinais negativos, e apenas livros (LIV) apresentou associação estatística significativa, porém negativa. Logo, há uma associação inversa entre a produção de livros e capítulo de livros por um lado, e os depósitos de patentes por outro. Assim como o resultado da variável senioridade, constata-se a não associação da produção de artigos e da participação em congressos na produção de depósitos de patente.

Em relação ao indicador de impacto econômico, apenas a produção artística (ARTE) não apresentou nível de significância, o que era esperado, visto que existem formas de proteção específicas para esse tipo de conhecimento, como desenho industrial e direito autoral. As variáveis produto tecnológico (PRO) e produção técnica (TEC) apresentaram sinal positivo e nível de significância de 1\% e 5\%, respectivamente. Quanto à variável de impacto social (JOR), seu resultado apresentou sinal negativo e foi significativa ao nível de 10\%, indicando que há associação inversa entre a produção de textos em jornais e revistas e a produção de patentes. Esse dado pode significar que os pesquisadores com maior produção de patentes possuem menor propensão a divulgar seus resultados de pesquisa em meios de comunicação não científicos. 
Tabela 1

Determinantes da produção de patentes dos pesquisadores mineiros.

\begin{tabular}{|c|c|c|c|}
\hline \multirow[t]{2}{*}{ Variáveis } & \multicolumn{3}{|c|}{ Coeficientes } \\
\hline & $\begin{array}{l}\text { Dados empilhados } \\
\text { (MQO) }\end{array}$ & Efeito aleatório & Efeito fixo \\
\hline \multirow[t]{2}{*}{ Senioridade (SEN) } & 0,00401 * & 0,00413 ** & 0,000376 \\
\hline & $(0,00150)$ & $(0,00216)$ & $(0,00824)$ \\
\hline \multirow[t]{2}{*}{ Orientações pós (ORIP) } & 0,0719 * & 0,0556 * & $0,0394 * * *$ \\
\hline & $(0,0153)$ & $(0,0177)$ & $(0,0180)$ \\
\hline \multirow[t]{2}{*}{ Orientações graduação (ORI) } & $-0,00772$ & $-0,00644$ & $-0,00552$ \\
\hline & $(0,00742)$ & $(0,00782)$ & $(0,00808)$ \\
\hline \multirow[t]{2}{*}{ Livros (LIV) } & $-0,0338$ * & $-0,0225$ * & $-0,0108 * *$ \\
\hline & $(0,00688)$ & $(0,00737)$ & $(0,00585)$ \\
\hline \multirow[t]{2}{*}{ Congressos (CON) } & $-0,0110$ & $-0,00508$ & $-0,00131$ \\
\hline & $(0,0135)$ & $(0,0173)$ & $(0,0165)$ \\
\hline \multirow[t]{2}{*}{ Artigos (ART) } & 0,0198 & 0,0101 & $-0,00196$ \\
\hline & $(0,0172)$ & $(0,0226)$ & $(0,0219)$ \\
\hline \multirow[t]{2}{*}{ Artística (ARTE) } & $-0,00950$ * & $-0,0119 * *$ & $-0,0139$ \\
\hline & $(0,00360)$ & $(0,00702)$ & $(0,00946)$ \\
\hline \multirow[t]{2}{*}{ Produto tecnológico (PRO) } & 0,320 * & 0,305 * & 0,292 * \\
\hline & $(0,0775)$ & $(0,0768)$ & $(0,0768)$ \\
\hline \multirow[t]{2}{*}{ Produção técnica (TEC) } & 0,0463 * & $0,0569 * * *$ & $0,0693 * * *$ \\
\hline & $(0,0159)$ & $(0,0267)$ & $(0,0312)$ \\
\hline \multirow[t]{2}{*}{ Jornais (JOR) } & $-0,0166$ * & $-0,0148$ * & $-0,0117$ ** \\
\hline & $(0,00569)$ & $(0,00530)$ & $(0,00597)$ \\
\hline \multirow[t]{2}{*}{ Dummy 1995} & 0,00383 & 0,00388 & $-0,0181$ \\
\hline & $(0,0205)$ & $(0,0201)$ & $(0,0372)$ \\
\hline \multirow[t]{2}{*}{ Dummy 1996} & 0,0734 & 0,0737 & $0,0533 * *$ \\
\hline & $(0,0494)$ & $(0,0552)$ & $(0,0323)$ \\
\hline \multirow[t]{2}{*}{ Dummy 1997} & 0,138 & 0,138 & 0,119 * \\
\hline & $(0,0974)$ & $(0,0995)$ & $(0,0717)$ \\
\hline \multirow[t]{2}{*}{ Dummy 1998} & $-0,00542$ & $-0,00644$ & $-0,0250$ \\
\hline & $(0,0201)$ & $(0,0165)$ & $(0,0356)$ \\
\hline \multirow[t]{2}{*}{ Dummy 1999} & 0,0143 & 0,0130 & $-0,00379$ \\
\hline & $(0,0211)$ & $(0,0184)$ & $(0,0295)$ \\
\hline \multirow[t]{2}{*}{ Dummy 2000} & $-0,00670$ & $-0,00912$ & $-0,0252$ \\
\hline & $(0,0189)$ & $(0,0169)$ & $(0,0268)$ \\
\hline \multirow[t]{2}{*}{ Dummy 2001} & 0,00346 & 0,00157 & $-0,0118$ \\
\hline & $(0,0216)$ & $(0,0166)$ & $(0,0255)$ \\
\hline \multirow[t]{2}{*}{ Dummy 2002} & 0,0157 & 0,0143 & 0,00417 \\
\hline & $(0,0223)$ & $(0,0177)$ & $(0,0238)$ \\
\hline \multirow[t]{2}{*}{ Dummy 2003} & 0,0153 & 0,0143 & 0,00724 \\
\hline & $(0,0219)$ & $(0,0203)$ & $(0,0241)$ \\
\hline \multirow[t]{2}{*}{ Dummy 2004} & $-0,00177$ & $-0,00204$ & $-0,01$ \\
\hline & $(0,0242)$ & $(0,0209)$ & $(0,0223)$ \\
\hline \multirow[t]{2}{*}{ Dummy 2006} & $-0,0161$ & $-0,0135$ & $-0,00762$ \\
\hline & $(0,0261)$ & $(0,0232)$ & $(0,0227)$ \\
\hline \multirow[t]{2}{*}{ Dummy 2007} & 0,0220 & 0,0272 & 0,0389 \\
\hline & $(0,0353)$ & $(0,0371)$ & $-(0,0362)$ \\
\hline
\end{tabular}

(continua) 
Tabela 1 (continuação)

\begin{tabular}{|c|c|c|c|}
\hline \multirow[t]{2}{*}{ Variáveis } & \multicolumn{3}{|c|}{ Coeficientes } \\
\hline & $\begin{array}{l}\text { Dados empilhados } \\
\text { (MQO) }\end{array}$ & Efeito aleatório & Efeito fixo \\
\hline \multirow[t]{2}{*}{ Dummy 2008} & 0,0229 & 0,0300 & 0,0470 \\
\hline & $(0,0355)$ & $(0,0281)$ & $(0,0299)$ \\
\hline \multirow[t]{2}{*}{ Dummy 2009} & $-0,0206$ & 0,0131 & 0,00813 \\
\hline & $(0,0292)$ & $(0,0306)$ & $(0,0378)$ \\
\hline \multirow[t]{2}{*}{ Dummy 2010} & $0,153 *$ & $0,163 *$ & $0,191 *$ \\
\hline & $(0,0439)$ & $(0,0472)$ & $(0,0539)$ \\
\hline \multirow[t]{2}{*}{ Dummy 2011} & $0,0812 * *$ & $0,0966 * \star *$ & $0,133 * \star \star$ \\
\hline & $(0,0447)$ & $(0,0435)$ & $(0,0567)$ \\
\hline \multirow[t]{2}{*}{ Dummy 2012} & $0,252 *$ & 0,269 * & $0,312 *$ \\
\hline & $(0,0611)$ & $(0,0548)$ & $(0,0701)$ \\
\hline \multirow[t]{2}{*}{ Dummy 2013} & 0,194 * & $0,214 *$ & $0,262 *$ \\
\hline & $(0,0541)$ & $(0,0599)$ & $(0,0859)$ \\
\hline \multirow[t]{2}{*}{ Dummy 2014} & 0,00302 & 0,0239 & 0,0765 \\
\hline & $(0,0427)$ & $(0,0505)$ & $(0,0852)$ \\
\hline \multirow[t]{2}{*}{ Dummy 2015} & $-0,159$ * & $-0,134$ * & $-0,0736$ \\
\hline & $(0,0311)$ & $(0,0486)$ & $(0,0957)$ \\
\hline \multirow[t]{2}{*}{ Dummy 2016} & $-0,113$ * & $-0,0997$ * & $-0,0516$ \\
\hline & $(0,0264)$ & $(0,0383)$ & $(0,0974)$ \\
\hline \multirow[t]{2}{*}{ Constante } & $-0,0666$ * & $-0,0737$ * & $-0,0511$ \\
\hline & $(0,0220)$ & $(0,0249)$ & $(0,0591)$ \\
\hline Observações & 17,160 & 17,160 & 17,160 \\
\hline Hausman & & 112,6 * & \\
\hline
\end{tabular}

MQO: mínimos quadrados ordinários.

Fonte: elaboração própria.

Notas: (1) encontram-se, entre parênteses, as estatísticas t e z (no caso dos efeitos aleatórios); (2) níveis de significância:

* Significativo a 1\%; ** Significativo a 10\%; *** Significativo a 5\%; (3) foram analisados os pesquisadores das

universidades federais mineiras com projetos aprovados no Edital Demanda Universal/FAPEMIG (2006-2015); (4) 2005 é

a dummy de referência.

Ao analisar as dummies de tempo, os anos de 1996, 1997, 2010, 2011, 2012 e 2013 apresentaram associação positiva, o que significa que, nesses anos, a produção de patentes foi superior à produção de 2005, ano de referência. Os anos de 2010, 2012 e 2013 foram os que apresentaram maior nível de significância (nível de 1\%).

\section{Discussão}

Apesar de recente, nota-se que a pesquisa acadêmica passou a ter relevância econômica, e transformá-la em inovação tornou-se um objetivo de governo, tanto na esfera federal quanto estadual. Com uma infraestrutura de conhecimento quase integralmente criada no século XX, os recentes avanços da produção de conhecimento científico e tecnológico demonstram o impacto das políticas públicas para a estruturação de um sistema de inovação mineiro, o que é confirmado pelos resultados apresentados. Há indícios de que as políticas públicas de CT\&I, representadas pelas dummies de tempo, apresentaram associação estatística positiva com os indicadores de produção científica e tecnológica usados neste artigo, além de indicarem a importância das habilidades individuais dos pesquisadores na questão do patenteamento.

A associação entre depósitos de patentes e orientações de pós-graduação pode ser um reflexo do Plano Nacional de Pós-Graduação (PNPG) 2011-2020, no qual seus objetivos foram baseados em 
tendências inovadoras e produtivistas, voltadas para o mercado e o desenvolvimento econômico 38 . Para o PNPG, a pesquisa é considerada ferramenta estratégica para o desenvolvimento econômico, assim como preconiza o modelo da Tríplice Hélice.

Ao analisar o Currículo Lattes dos pesquisadores que possuem produtos tecnológicos e patentes, foi observado que, em alguns casos, ambos contêm a mesma informação, o que pode justificar tal associação com os depósitos de patentes. Segundo dados disponíveis no Portal Brasil 39, a inserção da aba patentes no Lattes é recente. Logo, é provável que, antes de 2012, os registros de patentes fossem inseridos no currículo como produtos tecnológicos. Com relação à produção tecnológica, o resultado mostra a relevância da prestação de serviços e da conclusão de projetos, o que também pode indicar a importância da captação de recursos para a produção de depósitos de patentes. Sendo assim, há de se pensar na alocação de incentivos financeiros substancialmente maiores do que os disponibilizados atualmente, visto que inovação se tornou estratégica nacional.

Em contrapartida, a experiência acadêmica e o conhecimento científico (artigos) não apresentaram associação estatística, apontando para um possível conflito entre produção científica e tecnológica.

Esperava-se que a experiência científica fosse significante e positiva para a produção de depósitos de patentes, devido ao caráter cumulativo, sistêmico e idiossincrático do processo de aprendizagem, necessário para a produção de conhecimento. Contudo, devido ao seu caráter embrionário, é possível que o aprendizado necessário para a operação dos processos de inovação (gestão da inovação) ainda não tenha se consolidado, o que pode refletir uma melhor aceitação de jovens pesquisadores para a produção de patentes em detrimento da produção científica. Outras razões são provenientes da literatura internacional. É possível que os pesquisadores de Ciências da Vida, que sejam mais voltados para publicação de artigos, estejam trabalhando em áreas de ciência básica, com menor aplicação comercial dos resultados, e que haja um conflito de tempo, pois, quanto mais acadêmica a pesquisa, menor tempo pode restar para pensar em aplicações industriais, como ocorrido na área de ciências dos materiais, na Itália 17.

No que tange às dummies de tempo, o período de 2010 a 2013 apresentou produção de depósitos de patentes superior a 2005, sendo que, no início dos anos 2000, o estímulo à inovação passou a ter maior amplitude, culminando na criação dos fundos setoriais e na aprovação das Leis de Inovação em 2004 e do Bem em 2005. Ao analisar os anos de 1996 e 1997, que também apresentaram associação positiva, verifica-se a criação da Lei no 9.279, Lei de Propriedade Industrial 40, que passou a vigorar no ano de 1996. Além dessas políticas, ao analisar o Documento de Área, referente a Medicina I, da Coordenação de Aperfeiçoamento de Pessoal de Nível Superior (Capes), percebe-se uma mudança com relação às patentes nos triênios 2007-2009 e 2010-2012. No período 2007-2009, os depósitos de patentes não constavam como quesito de avaliação das publicações qualificadas do Programa por docente permanente 41 . Por outro lado, no período de 2010-2012, as produções do estrato B1 poderão ser substituídas por patentes publicadas no Web of Knowledge, particularmente no Derwent Innovations Index 42.

Embora seja possível perceber uma evolução quantitativa da produção de conhecimento e de patentes, também é cogente avaliar o direcionamento da política de fomento e regulação, se está baseado em mecanismos de controle ou de feedback seletivos que permitem o desenvolvimento do conhecimento científico e tecnológico. Em longo prazo, mecanismos de controle podem se tornar nocivos, ao formatar o perfil docente às metas da produtividade acadêmico-econômica. Outra preocupação é quando os esforços institucionais para patenteamento, embora crescentes em universidades, como no caso europeu, não produzem significativos retornos financeiros provenientes de licenciamento 43 .

No que tange ao papel das universidades, por possuírem o insumo principal da economia do conhecimento, o modelo da Tríplice Hélice apresenta-se no cerne do processo inovativo. Além de ensino e pesquisa, é necessário que as universidades, em termos práticos, incorporem a missão de empreendedoras. O empreendedorismo acadêmico deve ser visto como um passo natural no processo evolutivo e cumulativo das universidades, assim como na primeira revolução acadêmica, que agregou a pesquisa como segunda missão. Ao incorporar múltiplas missões, a universidade empreendedora deve não apenas criar mecanismos de estímulos isolados, mas desenvolver culturalmente esse novo paradigma de inovação. Para isso, ensino, pesquisa e inovação devem estar interconectados, de forma a permitir a concepção de uma totalidade, um sistema. Com relação ao ensino, isso pode ser observado pela relevância das orientações em pós-graduação para a produção de patentes. Contudo, 
o conhecimento científico, medido principalmente em artigos científicos, não impactou a produção tecnológica, o que mostra um descompasso na relação entre C\&T na área de saúde, hipótese já defendida no sistema de inovação da área de saúde no Brasil, também medida a partir de dados de artigos científicos e patentes 44 .

É clara a necessidade de desenvolver uma visão crítica do objetivo social da universidade e repensar o seu modelo. No entanto, é preciso entender o contexto da economia globalizada, a padronização do modo de produção acadêmica e o consequente resultado de suas pesquisas, para se evitar o desenvolvimento de uma massa social acrítica e formatada. A verdadeira batalha no mundo hoje não é apenas tecnológica e econômica, mas é de valores. Está relacionada com a evolução da espécie humana, em um novo cenário. A janela de oportunidade que se abre é a da educação. Não é a pautada em conteúdo (informação), mas a que privilegia a formação e a transformação do sujeito como agente de mudança social. Nesse sentido, a universidade empreendedora pode ser um respiro para a construção dessa sociedade do conhecimento, ao resgatar e recriar a sua primeira missão: o ensino.

Em termos metodológicos, este estudo limita-se a medir produção de conhecimento útil economicamente por patentes, sendo que, sobretudo na sociedade do conhecimento, valor econômico pode ser produzido por conhecimento não patenteável. Além disso, o estudo ressente-se de outras informações individuais que poderiam trazer resultados mais detalhados sobre o incentivo a patentear, como informações qualitativas sobre a posição do pesquisador em relação a retornos pecuniários e não pecuniários, importância das patentes para obtenção de financiamento para pesquisas e características de suas equipes de investigação. $\mathrm{O}$ estudo também se limita a uma área de conhecimento, que é o campo das Ciências da Vida. Evidências de outras áreas de conhecimento, até mesmo com maior propensão a patenteamento, precisam ser produzidas para fins de comparação.

\section{Conclusão}

O potencial de inovação e desenvolvimento econômico, em uma economia do conhecimento, encontra-se no papel de liderança da universidade e na hibridação proveniente da interação entre empresas, universidades e governos, capaz de gerar novos formatos institucionais e sociais para a produção, a transferência e a aplicação do conhecimento 45 . Essa visão engloba não só a destruição criativa que aparece como uma inovação dinâmica natural 3 , mas também a renovação que surge dentro de cada uma das três esferas institucionais.

Na tentativa de diminuir o hiato tecnológico, o modelo da Tríplice Hélice tem sido utilizado como norteador de políticas públicas de estímulo à inovação no Brasil, principalmente após 2004, com a criação da Lei de Inovação.

Como sugere o modelo teórico da Tríplice Hélice I, o sistema de inovação mineiro demonstra os esforços governamentais, mesmo que ainda incipientes, na tentativa de promover avanço tecnológico no Estado. Contudo, é necessário caminhar para a Tríplice Hélice III e entender que inovação não é um processo linear, mas o resultado de um conjunto complexo de relações entre os atores envolvidos na produção, na distribuição e na aplicação de vários tipos de conhecimento. As universidades precisam repensar o seu modelo (ensino e pesquisa) para assumir a sua missão de intervenientes ativos no processo de desenvolvimento econômico. De forma similar, o Estado precisa repensar o seu papel, estimular a liderança das universidades e criar uma infraestrutura de apoio. Para atuar em uma Economia do Conhecimento, há a necessidade de se despir dos velhos hábitos e recriar novos modelos de gestão. 


\section{Colaboradores}

I. Cóser, M. A. F. Brandão, N. R. B. Raposo e E. Gonçalves contribuíram na concepção, do projeto, análise e interpretação dos dados; na redação do artigo, revisão crítica relevante do conteúdo intelectual; na aprovação final da versão a ser publicada; e são responsáveis por todos os aspectos do trabalho, na garantia de exatidão e integridade de qualquer parte da obra.

\section{Agradecimentos}

Gostaríamos de agradecer à Universidade Federal de Juiz de Fora pela concessão de bolsa, imprescindível para a execução do projeto.

\section{Referências}

1. Pinch T, Bijker W. The social construction of facts and artefacts: or how the sociology of science and the sociology of technology might benefit each other. Soc Stud Sci 1984; 4:399441.

2. Perez C. Technological change and opportunities for development as a moving target. CEPAL Review 2001; (75):109-30.

3. Scumpeter J. Capitalismo, socialismo e democracia. Rio de Janeiro: Editora Fundo de Cultura; 1961

4. Perez C. Technological revolutions and techno-economic paradigms. (Working Papers in Technology Governance and Economic Dynamics; 2009). http://technologygovernance. $\mathrm{eu} /$ files/main/2009070708552121.pdf (acessado em 26/Fev/2017).

5. Papenhausen C. Causal mechanisms of long waves. Futures 2008; 40:788-94.

6. Lundvall B-Å. National systems of innovation: towards a theory of innovation and interactive learning. London: Pinter; 1992.

7. Nelson R, Nelson K. Technology, institutions, and innovation systems. Res Policy 2002; 31:265-72.

8. Cassiolato JE, Lastres HMM. Sistemas de inovação: políticas e perspectivas. Parcerias Estratégicas 2000; 8:237-55.

9. Johnson B, Lorenz E, Lundvall B-Å. Why all this fuss about codified and tacit knowledge? Industrial and Corporate Change 2002; 11:245-62.

10. Lundvall B-Å, Johnson B. The learning economy. Journal of Industry Studies 1994; (2):2342.

11. Klevorick AK, Levin RC, Nelson RR, Winter SG. On the sources and significance of interindustry differences in technological opportunities. Res Policy 1995; 24:185-205.

12. Etzkowitz H, Leydesdorff L. Universities and the global knowledge economy: a Triple Helix of university-industry-government relations. London: Cassell Academic; 1997.

13. Etzkowitz H, Leydesdorff L. The dynamics of innovation: from national systems and model 2 to a triple helix of university-industry-government relations. Res Policy 2000; 29:109-23.

14. Nagaoka S, Motohashi K, Goto A. Patent statistics as an innovation indicator. In: Hall $\mathrm{BH}$, Rosenberg N, editors. Handbook of the economics of innovation. v. 2. Amsterdam: Elsevier; 2010. p. 1083-127.

15. Acs ZJ, Audretsch DB. Innovation in large and small firms: an empirical analysis. Am Econ Rev 1989; 78:678-89.

16. Schettino F, Sterlacchini A, Venturini F. Inventive productivity and patent quality: evidence from Italian inventors. J Policy Model 2013; 35:1043-56.

17. Calderini M, Franzoni C, Vezzulli A. If star scientists do not patent: the effect of productivity, basicness and impact on the decision to patent in the academic world. Res Policy 2007; 36:303-19. 
18. Gurmu S, Black GC, Stephan PE. The knowledge production function for university patenting. Econ Inq 2010; 48:192-213.

19. Baldini N, Grimaldi R, Sobrero M. To patent or not to patent? A survey of Italian inventors on motivations, incentives, and obstacles to university patenting. Scientometrics 2007 ; 70:333-54.

20. Leydesdorff L, Ivanova I. "Open innovation" and "triple helix" models of innovation: can synergy in innovation systems be measured? Journal of Open Innovation: Technology, Market, and Complexity 2016; 2:11.

21. Etzkowitz H, Viale R. Polyvalent knowledge and the entrepreneurial university: a third academic revolution? Crit Sociol (Eugene) 2010; 36:595-609.

22. Tsai W. Knowledge transfer in intraorganizational networks: effects of network position and absorptive capacity on business unit innovation and performance. Acad Manage J 2001; 44:996-1004.

23. Burgos-Mascarell A, Ribeiro-Soriano D, Martínez-López M. Dystopia deconstructed: applying the triple helix model to a failed utopia. J Bus Res 2016; 69:1845-50.

24. Mazzucato M, Penna C. The Brazilian innovation system: a mission-oriented policy proposal. Brasília: Centro de Gestão e Estudos Estratégicos; 2016.

25. Freeman C, Perez C. Structural crises of adjustment business cycles and investment behaviour. In: Dosi G, Freeman C, Nelson R, Silverberg $G$, Soete $L$, editors. Technical change and economic theory. London: Pinter; 1988. p. 38-66.

26. Leydesdorff $\mathrm{L}$. The communication of meaning and the structuration of expectations: giddens'"structuration theory" and Luhmann's "self-organization". J Am Soc Inf Sci Technol 2010; 61:2138-50.

27. Koeller P. Política Nacional de Inovação no Brasil: releitura das estratégias do período 1995-2006 [Tese de Doutorado]. Rio de Janeiro: Instituto de Economia, Universidade Federal do Rio de Janeiro; 2007.

28. Morais JM. Políticas de apoio financeiro à inovação tecnológica: avaliação dos programas MCT/FINEP para empresas de pequeno porte. Brasília: Instituto de Pesquisa Econômica Aplicada; 2007. (Texto para Discussão, 1296).

29. Brasil. Lei Federal no 10.973, de 2 de dezembro de 2004. Dispõe sobre incentivos à inovação e à pesquisa científica e tecnológica no ambiente produtivo e dá outras providências. Diário Oficial da União 2004, 3 dez.

30. Arbix G, Consoni F. Inovar para transformar a universidade brasileira. Rev Bras Ciênc Soc 2011; 26:205-24.

31. Secretaria de Estado do Planejamento e Gestão de Minas Gerais. Plano Mineiro de Desenvolvimento Integrado (PMDI) 2007-2023. Belo Horizonte: Secretaria de Estado do Planejamento e Gestão de Minas Gerais; 2007.
32. Coser I, Gonçalves E. Instrumentos de apoio financeiro para parques tecnológicos: a experiência de Minas Gerais. Revista de Economia 2011; 37:53-77.

33. Gonçalves E, Cóser I. O Programa de Incentivo à Inovação como mecanismo de fomento ao empreendedorismo acadêmico: a experiência da UFJF. Nova Economia 2014; 24:555-96.

34. Mena-Chalco JP, Cesar-Jr. RM. ScriptLattes: an open-source knowledge extraction system from the Lattes Platform. Journal of the Brazilian Computer Society 2009; 15:31-9.

35. Moed HF, Halevi G. Multidimensional assessment of scholarly research impact. J Assoc Inf Sci Technol 2015; 66:1988-2002.

36. Baltagi BH. Econometrics analysis of panel data. 2nd Ed. Chichester: Wiley \& Sons; 2001.

37. Marques LD. Modelos dinâmicos com dados em painel: revisão de literatura. Porto: Centro de Estudos Macroeconómicos e Previsão, Faculdade de Economia do Porto; 2000.

38. Coordenação de Aperfeiçoamento de Pessoal de Nível Superior. Plano Nacional de Pós-Graduação (PNPG) 2011-2020. http://www.capes. gov.br/plano-nacional-de-pos-graduacao (acessado em 02/Fev/2017).

39. Portal Brasil. Currículo Lattes passa por mudanças no sistema para impedir fraudes. http:// www.brasil.gov.br/ciencia-e-tecnologia/ 2012/07/curriculo-lattes-passa-por-mudan cas-no-sistema-para-impedir-fraudes (acessado em 26/Jan/2017).

40. Brasil. Lei n. 9.279, de 14 de maio de 1996. Regula direitos e obrigações relativos à propriedade industrial. Diário Oficial da União 1996; 15 mai.

41. Coordenação de Aperfeiçoamento de Pessoal de Nível Superior. Documento de área 2009. http://www.capes.gov.br/images/stories/do wnload/avaliacao/MED_I20nov09q.pdf (acessado em 09/Jan/2017).

42. Coordenação de Aperfeiçoamento de Pessoal de Nível Superior. Documento de área 2013. https://docs.google.com/viewer? $\mathrm{a}=\mathrm{v} \&$ pid= sites\&srcid=Y2FwZXMuZ292LmJyfHRyaW VuYWwtMjAxM3xneDoyYjkwNDllNDMzY zk2ZDlm (acessado em 09/Jan/2017).

43. Geuna A, Nesta LJJ. University patenting and its effects on academic research: the emerging European evidence. Res Policy 2006; 35:790807.

44. Chaves CV, Albuquerque EDME. Desconexão no sistema de inovação no setor saúde: uma avaliação preliminar do caso brasileiro a partir de estatísticas de patentes e artigos. Economia Aplicada 2006; 10:523-39.

45. Leydesdorff L, Etzkowitz H. The Triple Helix as a model for innovation studies. Sci Public Policy 1998; 25:195-203.

46. Fundação para a Ciência e a Tecnologia. Domínios científicos e áreas científicas, 2012. https://www.fct.pt/apoios/projectos/concursos/ 2012/docs/Dominios_e_Areas_Cientificas_ C2012.pdf (acessado em 04/Jan/2017). 


\section{Abstract}

In the 1990s, the development of microelectronics and communication technologies spawned the information technology paradigm, characterized by an intensity of information which, transformed into knowledge and innovation, plays a crucial role for development. In order for this to occur, countries need to build appropriate institutional arrangements. This led to the Triple Helix model for systems innovation, which views innovation and technical progress in a Knowledge Economy as the result of a complex set of relations between universities, companies, and government. In this context, the current study aims to identify the impact of knowledge production and public policies for the production of patent applications by life sciences and health sciences researchers affiliated with federal universities in the State of Minas Gerais, Brazil. The analysis covered the years 1995 to 2016 and used econometric techniques in panel data. According to the findings, educational factors (thesis supervision), economic factors (technological products and the provision of technological services), and political factors (legal framework, financial resources, and incentive policies) impact the production of patent applications by the selected researchers. The results reveal efforts by government, although incipient, in the attempt to promote a technological leap.

Scientific and Technical Activities; Information Technology; Patents; Innovation

\section{Resumen}

Durante la década de 1990, con el desarrollo de la microelectrónica y las tecnologías de la comunicación, surgió el paradigma de la tecnología de la información, caracterizado por la intensidad de información que, transformada en conocimiento e innovación, desempeña un papel crucial para el desarrollo. No obstante, para que esto ocurra, es necesario que los países construyan marcos institucionales apropiados. Por ello, surge el modelo de sistemas de innovación de Triple Hélice, que abarca innovación y progreso técnico en una Economía del Conocimiento, como resultado de un conjunto complejo de relaciones entre universidad, empresa y gobierno. En este contexto, el presente estudio tiene como objetivo de identificar el impacto de la producción de conocimiento y de las politicas públicas, con el fin de producir depósitos para patentes de investigadores, pertenecientes al campo científico de las Ciencias de la Vida y la Salud, en el ámbito de las universidades federales de Minas Gerais, Brasil. El análisis consideró el período de 1995 a 2016, y utilizó técnicas de econometría de panel de datos. Los resultados muestran que los factores educacionales (orientación durante el posgrado), económicos (productos tecnológicos y prestación de servicios tecnológicos) y políticos (marco legal, recursos financieros y políticas de estímulo) impactan en la producción de depósitos para patentes de los investigadores seleccionados. Mediante el análisis de los resultados, se pueden percibir los esfuerzos del gobierno, aunque sean incipientes, en la tentativa de promover el salto tecnológico.

Actividades Científicas y Tecnológicas; Tecnología de la Información; Patentes; Innovación
Recebido em 06/Jun/2017

Versão final reapresentada em 12/Dez/2017 Aprovado em 18/Dez/2017 